Relations industrielles

Industrial Relations

\title{
Le Code civil du Québec et la Loi sur les normes du travail : convergence ou divergence?
}

\section{Jean-Yves Brière}

Volume 49, numéro 1, 1994

URI : https://id.erudit.org/iderudit/050920ar

DOI : https://doi.org/10.7202/050920ar

Aller au sommaire du numéro

Éditeur(s)

Département des relations industrielles de l'Université Laval

ISSN

0034-379X (imprimé)

1703-8138 (numérique)

Découvrir la revue

Citer cet article

Brière, J.-Y. (1994). Le Code civil du Québec et la Loi sur les normes du travail : convergence ou divergence? Relations industrielles / Industrial Relations, 49(1), 104-132. https://doi.org/10.7202/050920ar

\section{Résumé de l'article}

Quelles seront les incidences du nouveau Code civil sur la Loi sur les normes du travail ? De même que sa réciproque, quel sera l'effet de la Loi sur les normes sur l'interprétation des dispositions du Code ? Cette relation synergique qui devra s'établir entre les textes des deux législations fait l'objet de la présente étude. Cette analyse est à la fois comparative et prospective. L'auteur a retenu six points de comparaison : la prééminence des textes, la notion de salarié, le service continu, la rupture du lien d'emploi, le certificat de travail et l'aliénation d'entreprise.
Tous droits réservés @ C Département des relations industrielles de l'Université Laval, 1994
Ce document est protégé par la loi sur le droit d'auteur. L'utilisation des services d'Érudit (y compris la reproduction) est assujettie à sa politique d'utilisation que vous pouvez consulter en ligne.

https://apropos.erudit.org/fr/usagers/politique-dutilisation/ 


\title{
Le Code civil du Québec et la Loi sur les normes du travail
}

\section{Convergence ou divergence?}

\author{
Jean-Yves Brière
}

Quelles seront les incidences du nouveau Code civil sur la Loi sur les normes du travail? De même que sa réciproque, quel sera l'effet de la Loi sur les normes sur l'interprétation des dispositions du Code? Cette relation synergique qui devra s'établir entre les textes des deux législations fait l'objet de la présente étude. Cette analyse est à la fois comparative et prospective. $L$ 'auteur a retenu six points de comparaison: la prééminence des textes, la notion de salarié, le service continu, la rupture du lien d'emploi, le certificat de travail et l'aliénation d'entreprise.

Souvent, notre langage ne correspond plus à la réalité des choses. « Notre vocabulaire, notre grammaire, sont habités de métaphores vides de sens, de figures de langage usées ${ }^{1}$ ". Ainsi, nous continuons à parler du lever et du coucher du soleil comme si les découvertes de Copernic sur le système solaire n'avaient pas remplacé le système de Ptolémée. Il en est de même pour le langage juridique. Parfois, les lois ne reflètent plus la réalité des choses. Ainsi, notre Code civil du Bas-Canada qui fut adopté en 1865 ne correspondait plus à la réalité de la société québécoise. Qu'il suffise de rappeler que le Code $n$ 'avait que quelques dispositions ${ }^{2}$ traitant du louage d'ouvrage. D'ailleurs, l'article 1665a) définit comme suit le contrat de travail : « Le louage d'ouvrage est un contrat par lequel le locateur s'engage à faire quelque chose pour le locataire moyennant un prix. "

Le Code visait particulièrement à régir les rapports entre les « locateurs » et les «domestiques», «serviteurs », « compagnons » et « journaliers». Ce

* BRIÉRE, J.-Y., avocat, chargé de cours à l'Université de Montréal.

1 Georges Steiner, Réelles présences, Paris, Les éditions Gallimard, 1991, p. 21.

2 Art. 1665a) à 1671 C.c.B.-C. 
vocabulaire préindustriel ne collait plus à notre société postindustrielle. Les tribunaux ont dû interpréter pour ne pas dire triturer les textes pour étendre leur portée aux autres types d'employés. Ainsi, l'article $1668 \mathrm{du}$ Code s'applique non seulement aux domestiques et serviteurs mais également à tout employé, quel que soit le poste qu'il détient dans la hiérarchie de l'entreprise ${ }^{3}$. Les exemples étaient nombreux pour justifier la nécessité de modifier le Code civil $d u$ Bas-Canada et ce, particulièrement en regard du droit du travail qui, avouons-le, évolue très rapidement. Qu'il suffise d'évoquer quelques situations pour comprendre l'urgence de la réforme :

- la mondialisation des marchés;

- le développement d'entreprises multinationales;

- le chômage chronique pour une couche de salariés ;

- la montée du niveau de scolarisation;

- la spécialisation des emplois;

- le virage technologique;

- les mutations qu'ont subies les notions d'entreprise et d'employeur;

- le développement accéléré du secteur tertiaire ${ }^{4}$ et des conséquences qui en découlent, telles le travail à temps partiel, le statut de pigiste ou encore, la précarité du travail;

- la boulimie législative en ce domaine : les lois particulières se sont multipliées ces dernières années.

À une nouvelle société doit correspondre un droit nouveau ${ }^{5}$. Notre propos portera sur les nouvelles dispositions du Code civil du Québec (L.R.Q. 1991, c. 164) ayant trait au contrat de travail. Plus particulièrement, nous tenterons de répondre à la question suivante : Quelles seront les implications du nouveau Code civil à l'égard de la Loi sur les normes du travail?

Nous procéderons à l'arrimage parfois difficile du Code civil du Québec et de la Loi sur les normes du travail. Nous tenterons de dégager les incidences

3 Columbia Builders Supplies Co. c. Barlette, [1967] B.R. 111.

4 En $1950,46,4 \%$ de la population active occupait un emploi dans le secteur tertiaire. Ce pourcentage est passé à $72,1 \%$ en 1990 . Voir : Fernand MORIN, « La négociation collective selon le modèle de 1944 est-elle périmée? ", La négociation collective du travail : adaptation ou disparition?, Actes du $48^{\circ}$ congrès des relations industrielles, Université Laval, Sainte-Foy, Les Presses de l'Université Laval, 1993, p. 23.

5 D'ailleurs, le Ministre de la justice considère que le Code civil :

« a voulu traduire, à l'aube du XXI" siècle, les mutations profondes qu'a connues la société québécoise depuis l'adoption, en 1866, du Code civil du Bas-Canada dans les rapports sociaux et familiaux, les valeurs, les connaissances, le contexte économique, la perspective nouvelle des rapports humains dans la société et une meilleure adéquation au présent. Mais cette réforme s'inscrit aussi dans la continuité du droit antérieur; elle en constitue le prolongement, la bonification, la consolidation. » (p. VI)

Voir: Gil Rémillard, "Commentaires du Ministre de la justice », Le Code civil du Québec, Publications du Québec, 1993. 
du nouveau Code sur cette importante loi du travail ${ }^{6}$ ainsi que sa réciproque, soit l'effet de la Loi sur les normes sur l'interprétation des dispositions pertinentes du Code. Car, croyons-nous, l'influence ne peut être à sens unique; il y a plutôt une relation synergique qui doit s'établir. En somme, nous aborderons la coexistence des règles particulières du Code civil en matière de contrat de travail et des règles de la Loi sur les normes du travail?.

Cette analyse empruntera une approche comparative et prospective. Une telle approche nous semble des plus pertinentes et ce, pour ces deux raisons :

- vérifier si l'intention des codificateurs et le texte législatif qui en résulte ne souffre pas trop de distorsion;

- tenter d'entrevoir quelle pourra être l'interprétation des nouvelles dispositions à la lumière de la jurisprudence élaborée sous la Loi sur les normes du travail.

Afin de réaliser notre objectif, nous avons retenu, un peu arbitrairement, six points de comparaison: la prééminence des textes, la notion de salarié, la notion de service continu, la rupture du lien d'emploi, le certificat de travail, l'aliénation d'entreprise.

Notre étude se fondera sur les décisions rendues par les organismes administratifs (Commissaire du travail, arbitre de grief) ainsi que par les tribunaux de droit commun. Ajoutons qu'en cette matière, il est parfois difficile de rester sur le strict terrain du droit pur. Voilà pourquoi nous vous proposons d'ajouter certaines critiques et quelques prises de position personnelles.

\section{LA PRÉÉMINENCE DES TEXTES}

Code civil du Québec Loi sur les normes du travail

Disposition préliminaire

Art. 93. Normes d'ordre public. Sous Le Code civil du Québec régit, en harmonie avec la Charte des droits et libertés de la personne et les principes généraux du droit, les personnes, les rapports entre les personnes, ainsi que les biens.

réserve d'une dérogation permise par la présente loi, les normes du travail contenues dans la présente loi et les règlements sont d'ordre public.

6 Qu'il nous suffise de rappeler que cette loi s'applique directement à 1,3 millions de personnes réparties dans 163000 entreprises. L'an dernier, la Commission a eu à traiter 21000 plaintes provenant des salariés. Voir: Commission des normes du travail, Rapport annuel 1991-1992, Québec, 39 p.

7 D'ailleurs, dans un document d'orientation, le Ministre de la justice précisait que l'interrogation principale en matière de contrat de travail concernait la coexistence des règles du Code civil et celles de la Loi sur les normes. Voir : Gil RÉMILLARD, « Présentation du projet de Code civil du Québec ", (1991) 22 R.G.D. 5-77, p. 42. 
Le code est constitué d'un ensemble de règles qui, en toutes matières auxquelles se rapportent la lettre, l'esprit ou l'objet de ses dispositions, établit, en termes exprès ou de façon implicite, le droit commun. En ces matières, il constitue le fondement des autres lois qui peuvent elles-mêmes ajouter au code ou y déroger.

Art. 8. On ne peut renoncer à l'exercice des droits civils que dans la mesure où le permet l'ordre public.

Art. 9. Dans l'exercice des droits civils, il peut être dérogé aux règles du présent code qui sont supplétives de volonté; il ne peut, cependant, être dérogé à celles qui intéressent l'ordre public.
Disposition nulle. Une disposition d'une convention ou d'un décret qui déroge à une norme du travail est nulle de plein droit.

Art. 94. Condition de travail plus avantageuse. Malgré l'article 93, une convention ou un décret peut avoir pour effet d'accorder à un salarié une condition de travail plus avantageuse qu'une norme prévue par la présente loi ou les règlements.

Poser la problématique de la prééminence des textes, c'est soulever la question des sources du droit. Le droit commun codifié sert d'assise aux lois, il est en quelque sorte le substrat des lois. Le Code civil, dans sa disposition préliminaire, exprime bien ceci en spécifiant «qu'il [le Code civil] constitue le fondement des autres Lois ". La même disposition précise que le Code régit, en harmonie avec la Charte des droits et libertés de la personne et les principes généraux du droit, les rapports entre les personnes. C'est dire que le législateur place au plus haut échelon le Code civil dans la hiérarchie des sources de droit. Devant un tel énoncé de principe, les règles du droit commun devraient apparaître comme la solution ultime à tout conflit de lois. Cependant, cette même disposition préliminaire précise que les autres lois peuvent elles-mêmes ajouter au Code ou y déroger. C'est dans un tel contexte que la prééminence du Code ou de la Loi sur les normes se pose!

Un auteur ${ }^{8}$ soutient que la loi particulière pourra primer sur la disposition du Code civil dans la mesure où le législateur autorise une dérogation explicite. En somme, il serait nécessaire que le législateur énonce de façon non équivoque que la loi particulière doit primer. La Loi sur les normes ne contient aucune disposition précisant qu'elle doit primer sur le Code civil ${ }^{9}$. Malgré ce silence, nous croyons que la loi particulière doit prévaloir en cas de conflit. En effet, la Loi sur les normes du travail n'est pas une simple loi du travail. Il ne faut jamais oublier que le législateur a décrété le caractère d'ordre public des

8 Fernand MORIN, « La convention collective sous le prisme du nouveau Code civil ", (1993) 53 R. du B. 283-316, p. 287.

9 Précisons que la Loi sur l'application de la réforme du Code civil (L.Q. 1992, c. 57) ne prévoit aucune modification à la Loi sur les normes du travail. 
normes qu'elle énonce (art. 93) ${ }^{10}$. De ce fait, il nous semble qu'en cas de conflit irréductible, la loi spécifique doit s'appliquer indépendamment du Code civil et ce, pour trois raisons :

- D'abord, en raison de l'article 8 du Code civil qui précise que l'on peut renoncer à l'exercice d'un droit civil dans la mesure où l'ordre public ne le prohibe pas. Les dispositions du Code civil ne sont pas toutes impératives mais certaines sont plutôt supplétives de volonté.

- Ensuite, parce que l'article 9 du Code civil stipule qu'il est possible de déroger aux règles du Code qui n'intéressent pas l'ordre public. De ce fait, nous retenons l'argument que le Code doit céder le pas à un texte qui est spécifiquement décrété être d'ordre public (art. 93 L.N.T.).

- Finalement, la Loi sur les normes, à titre de loi particulière, doit recevoir une interprétation large et libérale suivant l'art. 41 de la Loi d'interprétation, de façon à lui donner la plus grande portée possible ${ }^{11}$. Il nous semble donc qu'à ce titre elle doit supplanter les règles générales du droit. D'ailleurs, le Ministre de la justice semble partager ce point de vue. Dans un document d'orientation, il s'exprimait ainsi : "Actuellement, l'avantprojet ne comporte pas de règles qui entreraient en contradiction avec la $\mathrm{Lo} i$ sur les normes et si, sur certains points, ces règles n'étaient pas exactement les mêmes, en raison entre autres de leur généralité, le caractère supplétif des règles empêcherait l'existence de conflits réels ${ }^{12}$ ".

Précisons que cette question de la hiérarchie des lois n'est pas que purement académique. Qu'il suffise de comparer le texte de l'article 2094 et celui de l'article 82.1 de la Loi sur les normes du travail pour s'en rendre compte ${ }^{13}$. Ainsi, en vertu du droit commun, il est possible de résilier le contrat de travail d'un salarié, sans préavis, si l'employeur a un motif sérieux. Aux termes de l'article 82.1 de la Loi sur les normes du travail cela n'est possible que si le salarié a commis une faute grave. Bien qu'il faille admettre que la faute grave

10 Sur la notion d'ordre public, voir : Pierre LAPORTE, « Le caractère d'ordre public des dispositions de la Loi sur les normes du travail ", (1987) 42 Relat. ind. 398; Paul BerNARD, La notion d'ordre public du droit administratif, Paris, Librairie générale de droit et de jurisprudence, 1962, 286 p.; Antonio Perreault, "Ordre public et bonnes moeurs ", (1949) $9 R$. du B. 1.

11 Cette interprétation large et libérale qu'il faut donner à la Loi sur les normes du travail ne fait plus aucun doute aujourd'hui. D'ailleurs, la Cour suprême du Canada a réaffirmé clairement ce principe, voir : Gilles Lefebvre c. HOJ Industries Ltd et autre, [1992] 1 R.C.S. 986.

12 Gil RémillaRd, "Commentaires du Ministre de la justice", précité note 5, p. 42.

13 Art. 2094 du C.c.Q. : « Une partie peut, pour un motif sérieux, résilier unilatéralement et sans préavis le contrat de travail ». Art. 82.1 de la L.N.T. : « Salariés non visés. L'article 82 ne s'applique pas à l'égard d'un salarié : 1) qui ne justifie pas de trois mois de service continu; 2) dont le contrat pour une durée déterminée ou pour une entreprise déterminée expire; 3) qui a commis une faute grave; 4) dont la fin du contrat de travail ou la mise à pied résulte d'un cas fortuit ". 
soit un motif sérieux, il nous faut ajouter qu'un motif sérieux n'est pas nécessairement une faute grave ${ }^{14}$. Heureusement, les textes ne semblent pas inconciliables. Ils sont plutôt complémentaires. Il n'en demeure pas moins que la Loi sur les normes devrait prévaloir.

\section{LA NOTION DE SALARIÉ}

\section{Code civil du Québec}

Art. 2085. Le contrat de travail est celui par lequel une personne, le salarié, s'oblige, pour un temps limité et moyennant rémunération, à effectuer un travail sous la direction ou le contrôle d'une autre personne, l'employeur.
Loi sur les normes du travail

Art. $1\left(10^{\circ}\right)$. « salarié » : une personne qui travaille pour un employeur et qui a droit à un salaire; ce mot comprend en outre le travailleur partie à un contrat en vertu duquel : i. il s'oblige envers une personne à exécuter un travail déterminé dans le cadre et selon les méthodes et les moyens que cette personne détermine;

ii. il s'oblige à fournir, pour l'exécution du contrat, le matériel, l'équipement, les matières premières ou la marchandise choisis par cette personne, et à les utiliser de la façon qu'elle indique;

iii. il conserve, à titre de rémunération, le montant qui lui reste de la somme reçue conformément au contrat, après déduction des frais d'exécution de ce contrat;

Dans un premier temps, soulignons qu'il existe plusieurs similitudes entre l'article 2085 C.c.Q. et l'article $1\left(10^{\circ}\right)$ de la Loi sur les normes $d u$ travail:

- Aucune formalité particulière n'est requise pour la matérialisation du contrat de travail. Ainsi, un contrat écrit n'est pas nécessaire. Une entente verbale suffit.

- Le travailleur bénévole est exclu. Pour se qualifier à titre de salarié, les deux dispositions exigent une rémunération ${ }^{15}$.

14 Cette distinction entre faute grave et motif sérieux existe également en France :

« [..] la jurisprudence admet qu'une faute légère du salarié peut ne pas constituer une cause réelle et sérieuse de licenciement et qu'une faute du salarié peut constituer une cause réelle et sérieuse de licenciement sans être pour autant une faute grave. " (p. 107)

Voir : Yves Delamotte, Le droit du travail en pratique, Paris, Les éditions d'organisation, 1984, $327 \mathrm{p}$.

15 Sur la question de l'exigence d'une rémunération pour se qualifier à titre de salarié, voir : Guimont c. Lévesque, Beaubien, Geoffrion Inc., D.T.E. 91 T-610 (C.T.); Commission des 
- La rémunération peut prendre plusieurs formes (salaire, participation aux profits, avantages ayant une valeur pécuniaire ou encore, l'octroi de dividendes) ${ }^{16}$.

- Les deux textes n'excluent pas les représentants de l'employeur. Ainsi, les cadres de l'entreprise sont considérés comme des salariés. Cependant, le cadre supérieur ${ }^{17}$ est exclu de l'application de la Loi sur les normes (sauf en ce qui concerne certaines normes ${ }^{18}$ ) mais il sera un salarié au sens du Code civil. Il en est de même de l'étudiant qui travaille en vertu d'un programme d'initiation au travail (art. 3 , par. $5^{\circ}$ de la Loi sur les normes $d u$ travail).

- Il n'est pas nécessaire qu'il s'agisse d'un emploi à temps complet. L'individu qui travaille à temps partiel ou encore à la pige peut être considéré comme un salarié ${ }^{19}$.

normes du travail c. Cogan Wire Metal Products (1974) Ltd, J.E. 82-1139 (C.P.); La Commission du salaire minimum c. Zone de ski Mauricie Inc., [1980] C.P. 79.

16 La rémunération peut prendre différentes formes : - l'octroi de certains avantages : Commission des normes du travail c. Lemcovitz, D.T.E. 90T-1288 (C.Q.); - la participation aux bénéfices : Barcona Ltée c. Boisvert, D.T.E. 84T-841 (T.A.); - le versement de commissions : St-Gelais c. Cie de fiducie du Canada Le Permanent, D.T.E. 85T-362 (C.T.), Pietrykowski c. Cie de fiducie du Canada Le Permanent, D.T.E. 85T-723 (T.A.), Commission des normes du travail c. La Compagnie de fiducie Canada Permanent, [1985] C.P. 284.

17 Selon la jurisprudence des Commissaires du travail, par cadre supérieur il faut entendre les seules personnes qui relèvent directement du conseil d'administration. Pour être un cadre supérieur, il faut posséder un niveau décisionnel qui se situe à celui de l'élaboration des politiques de l'entreprise, de la planification stratégique ainsi que du choix de mesures correctives d'ensemble à appliquer en fonction des résultats recherchés. En somme, il s'agit d'une exclusion tout à fait minime par rapport à l'ensemble des cadres d'une entreprise. Sur cette question, voir : Dussault c. Luminaires Liliane Auger Ltée, D.T.E. 92T-1096 (C.T.); Gosselin c. Burotec ventes, services et locations Inc., [1992] C.T. 525; Spina c. E.M.C. Marbre et céramique européen (1985) Inc., [1992] C.T. 148; Household Finance Corp. of Canada c. Garon, [1992] C.T. 109; Lee - Sharrock c. Banca Nazionale del Lavoro of Canada, [1987] 3 C.F. 578 (C.A.); Chabot c. Plomberie Albert Paradis Inc., D.T.E. 93T-302 (C.T.); Burkec c. Canadian Fine Color Co., D.T.E. 93T-252 (C.T.); Dussault c. London Life, compagnie d'assurance-vie, D.T.E. 93T-866 (C.T); Pierre Forget c. Les conseillers en placement Pemp Inc., [1993] C.T. 377; Jean-Yves BRIËRE, " Principaux amendements à la Loi sur les normes du travail et jurisprudence récente et marquante ", Développements récents en droit du travail (1991), Cowansville, Les éditions Yvon Blais, 1991, p. 6-7.

18 Précisons que ce n'est que depuis 1990 (Loi modifiant la Loi sur les normes du travail, L.Q. 1990, c. 73) que l'art. 3, par. 6 de la Loi sur les normes exclut partiellement le cadre supérieur. Cependant, les normes suivantes s'appliqueront à eux : a) congés pour événements familiaux (uniquement les art. 81.1 à 81.17) et l'indemnité de congé annuel qui en résulte (art. 74, al. 2, 3 et 4); b) aux règlements relatifs au congé de maternité ou au congé parental (art. 89(6)); c) à la section de la loi portant sur l'effet des normes du travail (art. 93 à 97) à savoir, le caractère d'ordre public des normes du travail; d) les dispositions relatives à la retraite (art. 84.1); e) les recours visant l'exercice des normes ci-haut mentionnées (art. 98 à 135, 122.1, 123.1 et 139 à 147).

19 Sur le statut d'un pigiste voir: Syndicat des employés des Publications Québécor (C.S.N.) c. Publications Québecor, [1977] T.T. 46 (requête en évocation rejetée); Yves Thibault c. Les Publications Photo Police Inc., [1984] T.A. 55. 
- Le contrat de travail peut être à durée déterminée ou indéterminée. L'article 2086 C.c.Q. le précise expressément ${ }^{20}$.

- Sous les deux textes législatifs, trois éléments sont essentiels pour que la notion de salarié puisse recevoir application : une prestation de travail, une rémunération et un lien de subordination ${ }^{21}$.

- Il n'est pas nécessaire que le travail s'effectue chez l'employeur. Le travailleur à domicile peut être compris dans la définition de salarié ${ }^{22}$. Une déci$\operatorname{sion}^{23}$ s'est appuyée sur le libellé de l'article 2 L.N.T. qui précise que « La présente Loi s'applique au salarié quel que soit l'endroit où il exécute son travail [...] " pour considérer un travailleur à domicile comme un salarié. Bien qu'une telle disposition n'existe pas en vertu du Code civil, son statut de droit commun fait en sorte qu'il doit recevoir application et ce, peu importe l'endroit où s'exécute la prestation de travail.

Au-delà de ces similitudes, voyons maintenant les personnes comprises dans ces définitions. La définition de salarié contenue au paragraphe $10^{\circ} \mathrm{de}$ l'article 1 de la Loi sur les normes du travail vise deux catégories de personnes. D'abord, le salarié au sens classique du terme, c'est-à-dire celui qui travaille pour autrui, sous son contrôle. En somme, celui qui est subordonné juridiquement à son employeur ${ }^{24}$. La jurisprudence n'exige cependant pas un contrôle direct sur l'exécution de la prestation de travail. En effet, la spécialisation des tâches et l'apparition d'emploi d'ordre technique ou professionnel ont assoupli considérablement cette notion. Il suffit que l'employeur fixe le cadre général

20 Comment distinguer le contrat à durée déterminée de celui à durée indéterminée. La Cour d'appel, dans Commission des normes c. Campeau Corporation, [1989] R.J.Q. 2108, suggère le critère suivant :

« Je conclus donc sur ce premier aspect de la question que, pour décider si le contrat de travail est d'une durée déterminée ou non, il faut au départ se reporter dans le temps à l'étape préliminaire des relations entre l'employeur et l'employé, celle où le premier offre de l'emploi et le second offre ses services. C'est à ce moment que se noue le lien contractuel et que les parties conviennent de sa durée. Celle-ci sera déterminée si la date à laquelle le contrat prend fin est fixée à l'avance entre elles ou si l'échéance est sujette à l'arrivée d'un événement, par exemple la terminaison de l'ouvrage pour lequel l'embauche a eu lieu. " (p. 2115).

21 Commission des normes du travail du Québec c. Sécurité domiciliaire R.G. Inc., D.T.E. 89T-1066 (C.Q.); Commission des normes du travail du Québec c. Les Immeubles Terrabelle Inc. et al., [1989] R.J.Q. 1307 (C.Q.); Commission des normes du travail du Québec c. Pensionnat Académie Pasteur Inc., D.T.E. 93T-253 (C.Q.).

22 Il le sera dans la mesure où il existe un lien de subordination. Cependant, un individu qui n'est soumis à aucun contrôle, qui n'a aucun horaire de travail et pour lequel aucune déduction à la source n'est effectuée, ne peut se qualifier comme salarié; Commission des normes du travail $d u$ Québec c. Entretien sanitaire Waterville inc., D.T.E. 93T-479 (C.Q.).

23 Commission des normes du travail c. International Forums, [1985] C.P. 1.

24 Alexandre Bastien c. Gouvernement du Québec, [1984] T.T. 7; Commission des normes du travail c. Laiterie Perrette, D.T.E. 84T-761 (C.S.). 
du travail à exécuter et conserve la faculté de superviser l'exécution ${ }^{25}$ ou encore, la dépendance vis-à-vis l'employeur peut se manifester par le port d'un uniforme ou l'utilisation d'un véhicule identifié à l'employeur ${ }^{26}$. En second lieu, la définition comprend également l'entrepreneur « dépendant ", c'est-àdire celui qui travaille pour autrui dans un cadre et selon les méthodes et moyens déterminés par l'employeur mais qui peut exécuter sa prestation avec son matériel, ses équipements et les matières premières ou marchandises qu'il a lui-même fournis. L'entrepreneur dépendant sera considéré comme un salarié et ce, même s'il fournit ses propres outils et matières premières et ce, dans la mesure où il existe une subordination, c'est-à-dire une forme de contrôle, entre ce dernier et son donneur d'ouvrage ${ }^{27}$.

La première catégorie de salariés est clairement comprise à l'article 2085 C.c.Q. En effet, bien que cet article n'emploie pas l'expression « lien de subordination ", il précise néanmoins que le travail doit être fait sous la direction ou le contrôle de l'employeur. L'utilisation de ces deux mots reflète la réalité moderne de ce qu'est le lien de subordination. Ainsi, l'employeur peut superviser le travail à distance et ce, sans qu'il n'y ait un contrôle direct et immédiat. Sous cet aspect, l'article 2085 C.c.Q. doit être lu en conjonction avec l'art. 2099 C.c.Q. qui précise que lorsqu'il existe un contrat d'entreprise, il n'y a aucun lien de subordination qui existe entre l'entrepreneur et le client. Cette absence totale de lien de subordination caractérise le contrat d'entreprise, c'est dire que l'existence d'un tel lien, si infime soit-il, caractérisera le contrat de travail. Qu'en est-il toutefois de la seconde catégorie - l'entrepreneur dépendant ? L'article 2103 C.c.Q., qui porte sur le contrat d'entreprise dispose que : «l'entrepreneur ou le prestataire de services fournit les biens nécessaires à l'exécution du contrat à moins que les parties n'aient stipulé qu'il ne fournirait que son travail ». A première vue, il semble se dégager de la lecture de l'article 2103 C.c.Q. que généralement lorsqu'une personne fournit les biens nécessaires à l'exécution du contrat, on soit en présence d'un véritable contrat d'entreprise et non d'un contrat de travail. Traditionnellement, le fait d'être propriétaire des outils de production et d'avoir la possibilité de faire des profits ou d'encourir des pertes a été considéré comme un des éléments qui distinguent «l'entrepreneur » du « salarié ». Considérant ces éléments, certains pourraient prétendre que l'entrepreneur dépendant $n$ 'est pas compris dans le cadre de

25 Monique Couture-Thibault c. Pharmajan Inc., [1984] T.A. 326.

26 Produits Shell Canada Ltée c. Martin, D.T.E. 88T-260 (C.S.).

27 Cette notion “d'entrepreneur dépendant » découle des alinéas i) ii) et iii) du paragraphe 10 soit la définition du mot salarié. Précisons que « Même si les alinéas i, ii et iii du paragraphe 10 ne sont liés par un « et » ni par « ou » dans la version française - ce qui paraît une crreur de rédaction - la conjonction de coordination " and " les unit dans la version anglaise. On doit en conclure que les trois conditions sont cumulatives ". Commission des normes du travail du Québec c. Les Immeubles Terrabelle Inc. et al., [1989] R.J.Q. 1307, 1310 (C.Q.). 
l'art. 2085 C.c.Q. puisqu'il peut réaliser des profits ou encourir des pertes. Pour notre part, nous croyons plutôt qu'il faut considérer l'entrepreneur dépendant comme un salarié au sens du Code civil et ce, pour deux raisons :

- d'abord parce que ce dernier exerce habituellement son travail sous le contrôle du donneur d'ouvrage. De ce fait, il existe un lien de subordination qui fait en sorte que la notion de contrat d'entreprise doit être écartée (art. 2099 C.c.Q.);

- les notions de direction et de contrôle sont suffisamment souples pour s'adapter à la réalité de l'entrepreneur dépendant.

Bien que la jurisprudence sera appelée à le décider, nous croyons qu'il est logique de prétendre que l'entrepreneur dépendant au sens de la Loi sur les normes du travail est détenteur d'un véritable contrat de travail au sens de l'article 2085 C.c.Q. Évidemment, il restera toujours des situations limites où certains détails particuliers feront osciller la décision dans un sens ou dans l'autre.

Selon nous, les deux textes législatifs semblent viser la même réalité. De ce fait, il apparaît que la jurisprudence développée sous la Loi sur les normes du travail sera utile pour déterminer les critères permettant de distinguer le «salarié » de "l'entrepreneur indépendant ». Plusieurs éléments ou indices pourront servir à départager l'un de l'autre:

- l'absence d'horaire précis de travail ${ }^{28}$;

- la dépendance économique ${ }^{29}$;

- l'enregistrement d'une déclaration de raison sociale ${ }^{30}$;

- la propriété des outils ${ }^{31}$;

- le pouvoir d'embaucher de la main-d'ceuvre ${ }^{32}$;

- l'existence de déductions à la source ${ }^{33}$;

- le statut d'actionnaire ou d'administrateur ${ }^{34}$;

28 Commission des normes du travail du Québec c. Les Immeubles Terrabelle Inc. et al., [1989] R.J.Q. 1307 (C.Q.); Commission des normes du travail du Québec c. Lemcovitz, D.T.E. 90T-1288 (C.Q.); Commission des normes du travail du Québec c. Déco Plan Inc., D.T.E. 89T-299 (C.Q.).

29 Fillion c. Club de curling Riverbond d'Alma, [1988] T.A. 442; Pierre Lajoie c. MultiMarques Inc., D.T.E. 87T-160 (T.A.).

30 Commission des normes du travail c. Deschambeault, D.T.E. 83T-776 (C.P.).

31 Padveen c. London Life, cie d'assurance-vie, D.T.E. 84T-421 (T.A.).

32 Rosaire Mailhot c. Services d'approvisionneurs national Inc., [1983] T.A. 1038.

33 Commission des normes du travail du Québec c. Sécurité domiciliaire R G. Inc., D.T.E. 89T-1066 (C.Q.); Commission des normes du travail c. Marois, D.T.E. 86T-573 (C.P.).

34 Brault c. Balances Leduc et Thibeault Inc., D.T.E. 89T-911 (T.A.); F.R.I. Information Services Ltd c. Viateur Larouche et al., [1982] C.S. 742. Jugement porté en appel C.A.M. 500-09-001145-820. Appel rejeté le 23-09-83; Visionic Inc. c. Michaud, J.E. 82-50 (C.S.); Beaudoin c. Mount-Royal Film Corp., D.T.E. 87T-292 (T.A.); Public Idée c. Auclair, D.T.E. 92T-699 (T.T.). 
- le fait de pouvoir s'adjoindre une aide ${ }^{35}$;

- la possibilité de faire des profits ou d'encourir des pertes ${ }^{36}$;

- le fait de bénéficier des avantages fiscaux des travailleurs autonomes $^{37}$.

En terminant, soulignons deux ambiguïtés. D'abord, l'article 2085 C.c.Q. ne définit pas la notion d'employeur. Cet oubli est regrettable car la réalité socio-économique moderne fait qu'il est parfois très difficile d'identifier l'employeur véritable. Qu'il nous suffise d'évoquer quelques situations particulières :

- lorsqu'une personne paie le salarié et qu'une autre dirige son travail;

- lorsqu'une agence de placement procure à un employeur du personnel;

- lorsqu'une société en commandite regroupe plusieurs personnes morales;

- lorsqu'une personne morale se démembre en plusieurs corporations distinctes;

- lorsqu'un individu travaille pour un holding regroupant plusieurs employeurs.

Afin de rechercher le véritable employeur, les tribunaux de droit commun seront certes portés à $s$ 'inspirer de la définition du mot «employeur "

35 Routliffe c. St-Andrews Presbyterian Church, D.T.E. 92 T-922 (C.T.).

36 Commission des normes du travail c. André Beaurivage, [1981] C.P. 47; Sklar Peper Inc. c. Marcel Loiselle, [1988] T.A. 449; André Huot c. Le manoir Richelieu et Famille Dufour, Cap-aux-Pierres Inc., [1984] T.A. 696.

37 Dans une décision récente, la Cour d'appel mettait fin à une controverse jurisprudentielle sur cette question :

« Pour ma part, je suis d'opinion que le comportement d'une personne à l'endroit des autorités fiscales ne constitue pas un critère déterminant pour établir son statut en vertu de la Loi sur les normes du travail. Il n'est toutefois pas interdit de considérer ce facteur parmi les autres éléments de preuve pour réussir à qualifier adéquatement cette personne. Un tel facteur pourra cependant être de peu de valeur si les deux législations en cause sont à ce point dissemblables qu'il n'existe entre elles aucun point de comparaison entre les définitions qu'elles contiennent et les objectifs qu'elles poursuivent. Dans l'état actuel de la législation, l'interprétation des termes " salariés " et " entrepreneurs " sous les lois fiscales ne correspond pas à celle de la Loi sur les normes du travail, d'autant plus que ces lois n'obéissent pas aux même règles d'interprétation. Il se peut que certaines personnes essaient de tirer un profit indû de cette approche en tentant de profiter du meilleur des deux mondes. Ce n'est cependant pas ce qui est en cause. »

North American Automobile Associations Ltd c. Commission des normes du travail du Québec, D.T.E. 93T-429 (C.A.). Pour les décisions antérieures voir : Commission des normes du travail c. International Forums Inc., D.T.E. 85T-8 (C.P.); à l'effet contraire : Commission des normes du travail c. Frédérick Dickson and Co., J.E. 80-822 (C.P.); Commission du salaire minimum c. Viceroy Construction Co. Ltd, J.E. 78-293 (C.P.). 
contenue à la Loi sur les normes du travail ${ }^{38}$ et de la jurisprudence qui en découla $^{39}$. L'identification du véritable employeur prendra une importance particulière puisque contrairement à la Loi sur les normes du travail (art. 95), le Code civil ne prévoit pas de responsabilité solidaire entre l'employeur et les sous-entrepreneurs ou sous-traitants.

La seconde ambiguïté réside dans l'utilisation du mot " personne » à l'article 2085 C.c.Q. En certaines circonstances particulières, serait-il possible de prétendre que le mot «personne » comprend une personne morale? En somme, si pour les fins de son travail un individu doit créer une corporation, peut-il se qualifier comme salarié? Au premier abord, cette question peut sembler saugrenue. En effet, comment accepter l'idée qu'une corporation puisse décéder au sens de l'article 2093 C.c.Q. ou encore recevoir un certificat de travail (art. 2096 C.c.Q.) lors de la terminaison du contrat de travail ${ }^{40}$ ? Malgré ce fait, il n'en demeure pas moins que cette question sera sûrement soulevée devant les tribunaux car elle le fut en vertu de la Loi sur les normes du travail. En effet, la Cour d'appel s'est penchée sur cette problématique. Dans l'arrêt Dazé c. Messageries Dynamiques ${ }^{41}$, la Cour considère que l'on ne peut permettre à un

38 Le mot employeur est défini comme la personne qui fait effectuer un travail par un salarié. Cette définition plutôt laconique fait cependant ressortir l'élément essentiel pour situer la portée de la définition du mot salarié. Elle précise la nécessité du lien de subordination. La définition ne réfère pas nécessairement à une seule personne ou à une seule entité juridique. Elle vise le fournisseur de la prestation de travail. À l'occasion, il pourra s'agir de plusieurs entités juridiques distinctes qui seront considérées comme un seul et même employeur.

39 Voir notamment : a) Payeur différent : Bourret c. Motel Carillon Hôtel Inc., D.T.E. 84T-686 (T.A.); Commission des normes du travail c. Commission scolaire St-Exupéry, D.T.E. 86T-451 (C.P.). b) Nécessité d'un lien de subordination : Monique Couture-Thibault c. Pharmajan Inc., [1984] T.A. 326; Christophe c. Les sacs à mains Santé Ltée et Les Agences Derma Ltée, [1984] T.A. 553. c) La notion d'employeur n'est pas limitée à une entité juridique : Jean-Claude Lapierre c. Pavane Mayfair Ltée, [1985] T.A. 380; Bourret c. Motel Carillon Hôtel Inc., D.T.E. 84T-686 (T.A.). d) Personnalités juridiques distinctes : Aluminium Luc Fauteux c. Morel, D.T.E. 88T-113 (T.T.); Alexandre Bastien c. Gouvernement du Québec, [1984] T.T. 7. e) Intermédiaire entre l'employeur et le salarié : Trottier c. Charbonneau, D.T.E. 87 T-715 (T.T.).; Commission des normes du travail c. Immeubles Terrabelle Inc. et al, [1989] R.J.Q. 1307 (C.Q.). f) Agence de placement : Commission des normes du travail c. 2548-9576 Québec Inc., D.T.E. 92 T-1034 (C.Q.).

40 Cette contradiction n'est peut-être qu'apparente. En effet, le libellé de l'art. 303 C.c.Q. donne peut-être la solution :

«Art. 303 : Les personnes morales ont la capacité requise pour exercer leurs droits, et les dispositions du présent code relatives à l'exercice des droits civils par les personnes physiques leur sont applicables, compte tenu des adaptations nécessaires.

Elles n'ont d'autres incapacités que celles qui résultent de leur nature ou d'une disposition expresse de la Loi. »

41 D.T.E. 90 T-538 (C.A.).; Au même effet, voir : Vachon c. Prudentielle (La), Cie d'assurance Ltée, D.T.E. 88T-221 (T.A.). Requête en évocation rejetée : Vachon c. Martin, D.T.E. 88-606 (C.S.); Habitabec c. Cloutier, D.T.E. 90T-1266 (C.S.). Décision portée en appel C.A.M. 500-09-001254-902. 
individu de jouer sur deux tableaux, soit l'utilisation du véhicule corporatif lorsque cela lui convient, par exemple, à des fins fiscales et le mettre de côté lorsque le donneur d'ouvrage met fin au contrat. Cependant, il serait peut-être possible de soutenir la thèse inverse. Notons que le nouveau Code parle de personnes morales (art. 298 C.c.Q. et suiv.) et non plus de corporations (art. 352 et suiv. C.c.B.-C.). On pourrait donc tirer de ce texte l'argument à l'effet que le vocable "personne " comprend tout aussi bien une personne physique qu'une personne morale. D'ailleurs, le paragraphe $16^{\circ}$ de l'article 61 de la Loi d'interprétation ${ }^{42}$ ne stipule-t-il pas que : «le mot "personne" comprend les corporations et s'étend aux héritiers et représentants légaux, à moins que la loi ou les circonstances particulières du cas ne s'y opposent "?

Ce nouvel argument de texte relancera peut-être le débat particulièrement dans les cas où l'incorporation ne résulterait pas d'un choix volontaire de l'employé mais plutôt d'une exigence de l'employeur pour éviter l'application de la Loi sur les normes du travail ou encore le chapitre du Code civil portant sur le contrat de travail ${ }^{43}$. Un tel comportement de l'employeur contreviendrait à l'art. 6 C.c.Q. qui stipule que : « Toute personne est tenue d'exercer ses droits civils selon les exigences de la bonne foi ». Dans ce cas, il serait possible de soulever le voile corporatif. D'ailleurs, l'article 317 du Code civil semble élargir les cas où le voile corporatif peut être soulevé : «La personnalité juridique d'une personne morale ne peut être invoquée à l'encontre d'une personne de bonne foi, dès lors qu'on invoque cette personnalité pour masquer la fraude, l'abus de droit ou une contravention à une règle intéressant l'ordre public $»$.

Le texte précise expressément qu'il sera possible de soulever le voile corporatif dans un cas "d'abus de droit » ou d'une "contravention à une règle intéressant l'ordre public ». Cette théorie ne serait plus limitée aux seuls cas de fraude ${ }^{44}$. Ce même article 317 pourra également servir de fondement à un recours en dommages intenté par un salarié contre un dirigeant de la personne morale qui a procédé au congédiement dans la mesure où ce congédiement pourra être qualifié d'abus de droit de la part du tribunal. Ainsi, ce dirigeant ne pourra plus se réfugier derrière la personnalité juridique de l'entreprise pour éviter une condamnation personnelle.

42 L.R.Q., c. I-16.

43 Dans une décision récente, un Commissaire du travail laisse entendre que si l'incorporation est imposée par l'employeur, la solution juridique pourrait être d'aller au-delà du voile corporatif. Voir : Jacques c. Itoh \& Cie (Canada) Ltée, [1992] C.T. 518.

44 Dazé c. Messagerie Dynamiques, D.T.E. 90T-538 (C.A.). L'arrêt classique sur cette question est Salomon c. Salomon, [1897] A.C. 22. 
Comme on peut le constater, l'apport de la Loi sur les normes du travail et de sa jurisprudence pour les fins d'interprétation de l'article 2085 C.c.Q. sera important.

\section{LA NOTION DE SERVICE CONTINU}

Code civil du Québec

Art. 2086. Le contrat de travail est à durée déterminée ou indéterminée.

Art. 2090. Le contrat de travail est reconduit tacitement pour une durée indéterminée lorsque, après l'arrivée du terme, le salarié continue d'effectuer son travail durant cinq jours, sans opposition de la part de l'employeur.
Loi sur les normes du travail

Art. $1\left(12^{\circ}\right)$. «service continu »: la durée ininterrompue pendant laquelle le salarié est lié à l'employeur par un contrat de travail, même si l'exécution du travail a été interrompue sans qu'il y ait résiliation du contrat, et la période pendant laquelle se succèdent des contrats à durée déterminée sans une interruption qui, dans les circonstances, permette de conclure à un nonrenouvellement de contrat.

C'est l'article 2086 C.c.Q. qui stipule que le contrat de travail est à durée déterminée ou indéterminée. Cet article est complété par une autre disposition, soit l'article 2090 C.c.Q. qui décrète que le contrat à durée déterminée se métamorphose en contrat à durée indéterminée s'il est reconduit tacitement. De plus, il y fixe la règle temporelle de la tacite reconduction. En effet, le salarié qui continue d'effectuer son travail durant cinq jours après l'expiration de son contrat verra son contrat de travail transformé. Pour que la transmutation s'opère, il ne faut pas que son employeur se soit opposé à cette période supplémentaire. Le Code ne prévoit pas si cette opposition doit être expresse ou si elle peut s'inférer des faits. Pour notre part, nous croyons qu'elle doit être exprimée de façon suffisamment claire pour que le salarié sache que l'employeur ne requiert plus ses services au-delà du terme du contrat et qu'il doit quitter son emploi. La règle retenue par le législateur diffère de celle que la tendance jurisprudentielle majoritaire avait adoptée à l'effet que lors d'une tacite reconduction, le contrat devait être renouvelé aux mêmes conditions ${ }^{45}$.

Il est également intéressant de comparer la règle de l'article 2090 C.c.Q. à celle de la définition de "service continu " contenue à l'art. 1 , par. $12^{\circ}$ de la Loi sur les normes du travail. À son origine, la Loi sur les normes avait retenu la notion suivante : « la durée ininterrompue pendant laquelle le salarié est lié à l'employeur par un contrat de travail, même si l'exécution du travail a été interrompue sans qu'il y ait résiliation du contrat». 
Cette définition se heurta rapidement à la réalité des contrats à durée déterminée qui étaient successivement renouvelés par l'employeur. Ce dernier plaidant qu'il ne pouvait y avoir de service continu puisqu'il s'agissait de contrats distincts. Assez rapidement, la jurisprudence ${ }^{46}$ établit qu'il importe peu que la continuité de la relation contractuelle découle d'un seul contrat de travail à durée indéterminée, d'un contrat à durée déterminée qui fut reconduit ou de la succession de plusieurs contrats à durée déterminée dans la mesure où il n'y a pas eu interruption du lien d'emploi. Le texte législatif fut modifié en $1990^{47}$ pour entériner ce mouvement jurisprudentiel. Ainsi, il est maintenant clair qu'un salarié peut bénéficier du recours à l'encontre d'un congédiement sans cause juste et suffisante s'il bénéficie de trois ans de service continu qui découlent de la succession de différents contrats de travail à durée déterminée. Pour sa part, l'article 2090 C.c.Q. ne traite que de la tacite reconduction. Il sera donc possible pour un employeur d'éviter celle-ci en octroyant au salarié une succession de contrats à durée déterminée. Ainsi, les contrats à durée déterminée se suivront sans qu'il n'y ait de métamorphose en contrat à durée indéterminée. L'employeur évitera ainsi l'obligation de payer le délai de congé dû aux termes de l'article 2091. Une telle situation serait pour le moins bizarre puisqu'un salarié placé dans un tel cas et qui compterait au moins trois ans de service continu ne pourrait bénéficier d'un délai de congé en vertu du Code civil mais il pourrait s'en voir octroyer un par le Commissaire du travail suite à une plainte déposée sous l'article 124 de la Loi sur les normes du travail. Il est pour le moins curieux que le législateur n'ait pas su mieux harmoniser la règle de l'article 2090 C.c.Q. avec celle de la notion de service continu. Il nous semble que le législateur répète ici l'erreur qu'il avait commise en 1979 lors de la première version de la définition de service continu.

Dans un autre ordre de questionnement, la compréhension de la notion de service continu sera peut-être modifiée par l'effet indirect des nouvelles dispositions du Code civil. En effet, jusqu'à présent, la mise à pied pour manque de travail n'est pas considérée comme une rupture du lien d'emploi ${ }^{48}$. Sauf si cette mise à pied résulte d'une abolition de poste ${ }^{49}$. Ainsi, un salarié peut accumuler du service continu et ce, même s'il est en mise à pied temporaire. De ce fait, il faut en déduire que le contrat de travail subsiste durant cette période. Cette prétention sera peut-être plus difficile à soutenir compte tenu que le Code

46 Voir notamment: Commission scolaire Berthier-Nord-Joli c. Rita Beauséjour et Bernard Lefebvre, [1988] R.J.Q. 639 (C.A.); Mark C. Moore c. Compagnie Montréal Trust, [1988] R.J.Q. 2339 (C.A.); Chamberland c. Desnoyers, D.T.E. 90 T-993 (C.S.).

47 Loi modifiant la Loi sur les normes du travail, L.Q. 1990, c. 73.

48 Commission des normes du travail du Québec c. Studio Sylvain Dethioux Inc., D.T.E. 90T-934 (C.Q.); Ateliers Roland Gingras Inc. c. Yves Desrochers, [1987] T.A. 600; Labanowako c. Grands Magasins Towers Inc., S.E. 91-667 (C.Q.).

49 Ménard c. Optigo Ltée, D.T.E. 91 T-48 (T.A.). 
stipule expressément que l'employeur est tenu de permettre l'exécution de la prestation de travail du salarié (art. 2087 C.c.Q.). Lorsque l'employeur procède à une mise à pied, il viole cette obligation ${ }^{\text {s0 }}$ et de ce fait, ne serait-il pas possible de prétendre qu'il n'y a plus de contrat de travail puisqu'un des cocontractants ne respecte plus ses engagements ${ }^{51}$ ? Par voie de conséquence, s'il y a rupture du lien contractuel, il sera difficile de prétendre que le service continu peut s'accumuler. D'ailleurs, la Cour d'appel ${ }^{52}$ a déjà considéré que le fait de mettre un salarié en congé sans solde constituait une résiliation du contrat de travail : «Le refus de l'employeur de permettre à l'employé de fournir ses services et la cessation du paiement du salaire constituent une résiliation unilatérale, un licenciement sans préavis ${ }^{53}$ ".

\section{LA RUPTURE DU LIEN D'EMPLOI}

Code civil

Art. 2091. Chacune des parties à un contrat à durée indéterminée peut y mettre fin en donnant à l'autre un délai de congé.

Art. 2092. Le salarié ne peut renoncer au droit qu'il a d'obtenir une indemnité en réparation du préjudice qu'il subit, lorsque le délai de congé est insuffisant ou que la résiliation est faite de manière abusive.

Art. 2094. Une partie peut, pour un motif sérieux, résilier unilatéralement et sans préavis le contrat de travail.
Loi sur les normes du travail

Art. 82. Avis de fin de contrat. Un employeur doit donner un avis écrit à un salarié avant de mettre fin à son contrat de travail ou de le mettre à pied pour six mois ou plus.

Délais. Cet avis est d'une semaine si le salarié justifie de moins d'un an de service continu, de deux semaines s'il justifie d'un an à cinq ans de service continu, de quatre semaines s'il justifie de cinq à dix ans de service continu et de huit semaines s'il justifie de dix ans ou plus de service continu.

Nullité d'avis de cessation d'emploi. L'avis de cessation d'emploi donné à un salarié pendant la période où il a été mis à pied est nul, sauf dans le cas d'un emploi dont la durée n'excède habituellement pas six

50 Sauf si le salarié accepte cette mise à pied ou encore si la mise à pied est liée à la nature du travail (emploi saisonnier) et ainsi comprise dans le contrat de travail.

51 Cette prétention est d'autant plus vraisemblable qu'il n'est plus nécessaire de faire prononcer la résiliation du contrat de travail par voie judiciaire. En effet, l'article 1605 C.c.Q. prévoit que la résiliation d'un contrat peut avoir lieu sans poursuite judiciaire lorsque le débiteur est en demeure de plein droit d'exécuter son obligation. Sur la question de la demeure de plein droit, voir art. 1597 C.c.Q.

52 Surveyer, Nenniger et Chênevert Inc. c. Thomas, D.T.E. 89T-640.

53 Id., p. 4. 
mois à chaque année en raison de l'influence des saisons.

Droits acquis. Le présent article n'a pas pour effet de priver un salarié d'un droit qui lui est conféré par une autre loi.

Art. 83. Indemnité compensatrice. L'employeur qui ne donne pas l'avis prévu à l'article 82 ou qui donne un avis d'une durée insuffisante doit verser au salarié une indemnité compensatrice équivalente à son salaire habituel, sans tenir compte des heures supplémentaires, pour une période égale à celle de la durée ou de la durée résiduaire de l'avis auquel il avait droit.

Moment du versement. Cette indemnité doit être versée au moment de la cessation d'emploi ou de la mise à pied prévue pour plus de six mois ou à l'expiration d'un délai de six mois d'une mise à pied pour une durée indéterminée ou prévue pour une durée inférieure à six mois mais qui excède ce délai.

Salarié rémunéré à commission. L'indemnité du salarié principalement rémunéré à commission est établie à partir de la moyenne hebdomadaire de son salaire durant les périodes complètes de paie comprises dans les trois mois précédant sa cessation d'emploi ou sa mise à pied.

Art. 124. Plainte de congédiement. Le salarié qui justifie de trois ans de service continu dans une même entreprise et qui croit avoir été congédié sans une cause juste et suffisante peut soumettre sa plainte par écrit à la Commission ou la mettre à la poste à l'adresse de la Commission dans les 45 jours de son congédiement, sauf si une procédure de réparation, autre que le recours en dommagesintérêts, est prévue ailleurs dans la présente loi, dans une autre loi ou dans une convention. 
Défaut. Si la plainte est soumise dans ce délai au commissaire général du travail ou au ministre, le défaut de l'avoir soumise à la Commission ne peut être opposé au plaignant.

Plusieurs dispositions de la Loi sur les normes du travail ${ }^{54}$ peuvent être applicables lors de la rupture du lien d'emploi. Cependant, pour les fins de cette étude comparative, nous nous bornerons à analyser les articles ayant trait à l'avis de cessation d'emploi (art. 82, 82.1, 83 et 83.1 L.N.T.) ainsi qu'au recours à l'encontre d'un congédiement fait sans une cause juste et suffisante (art. 124 L.N.T.). Nous traiterons de ces dispositions en les mettant en parallèle avec les articles 2091 et 2094 du Code civil du Québec.

L'article 82 de la Loi sur les normes du travail précise qu'avant de mettre fin à un contrat de travail, l'employeur doit donner un avis écrit au salariés ${ }^{55}$. Cet avis doit être donné au salarié peu importe le motif de la rupture du lien d'emploi (licenciement ou congédiement). La durée de l'avis varie en fonction du nombre d'années de service continu du salariés ${ }^{56}$. A défaut de donner un tel avis ou encore de donner un avis suffisant, l'employeur doit verser au salarié une indemnité tenant lieu de préavis.

Cet avis préalable ou l'indemnité qui en tient lieu doit être distingué de l'indemnité de délai-congé qui peut être accordée par un Commissaire du travail suite à une plainte de congédiement sans cause juste et suffisante. En effet, le Commissaire du travail saisit d'une plainte sous l'article 124 qui conclut au bien-fondé de la plainte et à une impossibilité d'une ordonnance de réintégration, doit fixer l'indemnité tenant lieu de délai-congé qui est dû au salarié. Cette indemnité varie en fonction des mêmes critères que ceux qui furent établis par les tribunaux de droit commun ${ }^{57}$. La jurisprudence ${ }^{58}$ reconnait qu'il

54 Voir notamment les articles $82,82.1,83,83.1,83.2,84,84.1,122,122.1,122.2,123$ et 124 .

55 L'avis écrit s'applique également lors d'une mise à pied de plus de six mois.

56 De trois mois à moins d'un an de service = une semaine; $d$ 'un an à cinq ans de service $=$ deux semaines; de cinq ans à dix ans de service = quatre semaines; dix ans et plus de service $=$ huit semaines.

57 Voir notamment: Columbia Builders Supplies Co. c. Bartlett, [1967] B.R. 111; Jacques Jolicoeur c. Lithographie Montréal Ltée, [1982] C.S. 230; Steinberg's Limited c. Jean Lecompte, [1985] C.A. 223.

58 Liberty Mutual Insurance Co. c. Commission des normes du travail du Québec, D.T.E: 90T-872 (C.A.); Commission des normes du travail du Québec c. Compagnie de construction Lazar Inc., D.T.E. 92T-870 (C.Q.); Commission des normes du travail du Québec c. Turcotte, D.T.E. 88T-776 (C.P.); Commission des normes du travail du Québec c. Guide de Montréal-Nord Inc., D.T.E. 88T-863 (C.P.); Commission des normes du travail c. Les Placements Sogelon, [1985] 
est possible de cumuler le recours sous l'article 83 et une plainte sous l'article 124 , puisque les deux recours sont distincts et qu'ils visent des buts différents.

Pour sa part, le nouveau Code civil traite de la rupture du contrat de travail dans les trois articles suivants : 2091, 2092,2094. Le premier précise que les parties peuvent mettre fin au contrat de travail à durée indéterminée en donnant au cocontractant un délai de congé. Cette expression de délai de congé est nouvelle. Elle vise sûrement la même réalité que l'expression généralement reconnue en droit du travail qu'est le délai-congé. Par cet article, le législateur codifie la règle édictée par la jurisprudence à l'effet que l'employeur peut unilatéralement mettre fin au contrat à durée indéterminée, sans cause, à la condition de donner à son employé un délai-congé raisonnable. Le second article consacre le caractère d'ordre public de l'indemnité que représente le délai de congé. En effet, il décrète que l'on ne peut renoncer au droit d'obtenir une telle indemnité. En vertu de la Loi sur les normes, la jurisprudence a reconnu qu'une disposition contractuelle ne pouvait limiter le pouvoir de l'arbitre d'accorder un délai-congé aux termes de l'art. 124 L.N.T. ${ }^{59}$. Pour sa part, l'article 2094 C.c.Q. prévoit qu'il est possible de résilier le contrat de travail sans préavis si une partie dispose d'un motif sérieux.

Une constatation est à faire au niveau du vocabulaire utilisé. Ainsi, à l'article 2091, le législateur emploie deux expressions : " mettre fin » et « délai de congé » alors qu'à l'article 2094 C.c.Q., il utilise les expressions « résilier » et «préavis ». Ce manque d'uniformité de langage soulève deux questions :

- Y a-t-il une distinction entre la notion de délai de congé et celle de préavis? A titre d'exemple, ne pourrait-on pas prétendre que le préavis dont il est fait mention à l'article 2094 C.c.Q. est semblable à celui visé par les articles 82 et 83 de la Loi sur les normes du travail alors que le délai de congé de l'article 2091 C.c.Q. viserait le délaicongé accordé par le Commissaire aux termes de l'article 124 de la Loi sur les normes du travail. Comme nous l'avons souligné, la jurisprudence distingue l'avis de cessation d'emploi de l'article 82 L.N.T. et le délai-congé accordé en vertu de l'article 124 L.N.T. Une telle distinction ne pourrait-elle pas exister entre les articles 2091 C.c.Q. et 2094 C.c.Q.? Si une telle distinction existe, elle justifierait l'emploi du mot "sérieux " (art. 2094 C.c.Q.) plutôt que l'expression consacrée de «cause juste et suffisante». Ainsi, il serait possible d'éviter le préavis si une partie a un motif sérieux alors que pour éviter le

C.P. 151. À l'effet contraire : Commission des normes du travail du Québec c. Maheu et Noiseux Inc., D.T.E. 89T-229 (C.Q.).

59 Martin c. Compagnie d'assurances du Canada sur la vie, [1987] R.J.Q. 514 (C.A.); Zeller's Inc. c. Laurin, [1986] R.J.Q. 1864 (C.A.); Bilodeau c. Bata Industries Ltd, [1986] R.J.Q. 531 (C.A.); Holt Renfrew \& Co. c. Legendre, D.T.E. 87 T-85 (C.S.). 
délai-congé, il serait nécessaire d'avoir une cause juste et suffisante.

- Les expressions «mettre fin » et « résilier 》 visent-elles la même réalité ? Il est pour le moins curieux que le législateur ait fait usage de deux termes différents et ce, à trois articles de distance, pour parler de la rupture du lien d'emploi. S'agirait-il là d'un simple exercice de style?

La lecture conjuguée de tous ces articles nous pose les interrogations suivantes :

- Comment concilier le motif sérieux spécifié à l'article 2094 du Code et la faute grave prévue à l'article 82.1 de la Loi sur les normes $d u$ travail ? Ces deux expressions sont fort différentes. Le concept de faute grave fait référence à un comportement délinquant de la part du salarié et ce comportement doit être suffisamment grave pour exiger la rupture immédiate du lien d'emploi ${ }^{60}$. Pour sa part, le motif sérieux ne fait pas nécessairement référence à un comportement du salarié. Il vise plutôt à établir un certain "contrôle de qualité » du motif invoqué. Ainsi, par exemple, l'absentéisme d'un salarié n'est pas nécessairement une faute grave mais peut être un motif sérieux de congédiement ${ }^{61}$ ou encore l'obtention d'un emploi chez un compétiteur ne constitue pas une faute grave ${ }^{62}$ mais peut constituer un motif sérieux ${ }^{63}$.

- Comment également concilier le motif sérieux et la notion de cause juste et suffisante (art. 124 L.N.T.)? La notion de cause juste et suffisante est largement répandue en droit du travail et plusieurs lois y font allusion ${ }^{64}$. Cette notion recoupe deux éléments : un manquement de la part du salarié et une proportionnalité entre ce manquement et

60 Pour des exemples de faute grave, voir : Commission des normes du travail c. Centre de Pneu Papineau (1982) Inc., D.T.E. 87T-407 (C.P.); Jean-Pierre Brisson c. Société Sandwell Ltée, [1984] C.P. 88; Commission des normes du travail c. Brasserie la Houblonnière Inc., [1986] R.J.Q. 2887 (C.P.); Commission des normes du travail c. Investissements Trizek Limitée, [1985] C.P. 273; Commission des normes du travail c. Provigo (distribution) Inc., [1986] R.J.Q. 912 (C.P.); Commission des normes du travail c. Union Carbide du Canada Ltée, [1986] R.J.Q. 1498 (C.P.).

61 Commission des normes du travail c. Centre d'accueil Edmond Laurendeau, [1987] R.J.Q. 1449 (C.P.).

62 Commission des normes du travail c. Exterminations St-Michel Ltée, D.T.E. 88T-27 (C.P.).

63 Le professeur Bich considère pour sa part que ces deux expressions sont des synonymes. Voir : Marie-France BICH, "Le contrat de travail ", La réforme du Code civil, Sainte-Foy, Les Presses de 1'Université Laval, 1993, p. 779.

64 Art. 17 C.t.; art. 123 L.N.T.; art. 124 L.N.T.; art. 255 de la Loi sur les accidents du travail et les maladies professionnelles; art. 227 de la Loi sur la santé et la sécurité du travail; art. 47 de la Charte de la langue française. 
le congédiement imposées. Pour sa part, le motif sérieux fait également référence à un manquement du salarié, qu'il soit volontaire ou non, peu importe, mais il ne vise pas nécessairement la règle relative à la proportionnalité. Cette dernière notion (motif sérieux) nous semble plus restrictive et moins exigeante à l'égard de l'employeur. Ainsi, certains pourront prétendre avoir un motif sérieux et ce, même si le congédiement peut sembler une mesure trop sévère dans les circonstances.

- Le paiement d'une indemnité par l'employeur conformément à l'art. 82 L.N.T. constitue-t-il une fin de non recevoir à une action en réclamation d'un délai de congé basée sous l'art. 2091 C.c.Q.? La réponse à cette question ne devrait pas être différente de celle que la jurisprudence a élaborée en vertu de l'article 1668 C.c.B.-C. Après bien des hésitations ${ }^{66}$, la Cour d'appel ${ }^{67}$ confirmait le courant jurisprudentiel reconnaissant la subsistance du droit de réclamer un préavis d'une durée raisonnable eu égard à l'usage et aux critères dégagés par la jurisprudence et ce, indépendamment du paiement de l'avis de cessation d'emploi en vertu de l'article 82 L.N.T.

Ces quelques questions étant soulevées, il nous est maintenant possible de déplorer le peu de congruence entre les dispositions du Code et celles de la Loi sur les normes du travail lors de la rupture du lien d'emploi.

\section{LE CERTIFICAT DE TRAVAIL}

\section{Code civil du Québec}

Art. 2096. Lorsque le contrat prend fin, l'employeur doit fournir au salarié qui le demande un certificat de travail faisant état

\section{Loi sur les normes du travail}

Art. 84. Certificat de travail. À l'expiration du contrat de travail, un salarié peut exiger que son employeur lui délivre un certificat

65 Rodrique Blouin, « Notion de cause juste et suffisante en contexte de congédiement », (1981) $41 R$ du B. 807; Pierre LAPORTE, Le traité du recours à l'encontre d'un congédiement sans cause juste et suffisante, Montréal, Wilson et Lafleur, 1992, $576 \mathrm{p}$.

66 Certains jugements considéraient qu'un salarié ne pouvait réclamer un préavis en vertu de l'art. 1668 C.c.B.-C. s'il avait reçu un préavis conformément à l'article 82 L.N.T. Voir : Durant c. Radio Etchemin Inc., D.T.E. 88T-188 (C.S.); Vigeant c. Canadian Thermos Products Ltd, D.T.E. 88T-295 (C.S.). Par contre, d'autres décisions étaient à l'effet que l'art. 82 constituait une norme minimale et que les circonstances pouvaient justifier l'octroi d'une indemnité plus généreuse, voir: Robert Brown c. Goulon Capital Corporation, [1986] R.J.Q. (C.S.); Nyveen c. Russel Food Equipment Ltd, D.T.E. 88T-294 (C.S.).

67 Domtar c. St-Germain, [1991] R.J.Q. 1273. La Cour d'appel prit la même position eu égard au Code canadien du travail (art. 230 C.c.t.); voir : Transports Kingsway Ltée c. Laperrière, D.T.E. 93T-197 (C.A.). 
uniquement de la nature et de la durée de l'emploi et indiquant l'identité des parties. de travail faisant état exclusivement de la nature et de la durée de son emploi, du début et de la fin de l'exercice de ses fonctions ainsi que du nom et de l'adresse de l'employeur. Le certificat ne peut faire état de la qualité du travail ou de la conduite du salarié.

L'article 2096 C.c.Q. élève au rang d'obligation contractuelle le certificat de travail. Comme on le sait, le certificat de travail n'est pas inconnu dans notre droit puisqu'il est déjà prévu à l'article 84 de la Loi sur les normes $d u$ travail.

D'une lecture comparative des textes ${ }^{68}$, nous retenons les observations suivantes :

- Il importe peu que la résiliation du contrat de travail résulte de l'une ou de l'autre partie.

- La disposition s'applique indépendamment de la qualification (congédiement, licenciement, démission) de la rupture du lien d'emploi.

- Il n'y a obligation de l'employeur que dans la mesure où le salarié exige le certificat. Il faut, selon nous, une demande expresse du salarié à cet effet. On ne peut déduire du simple fait du congédiement que le salarié requiert implicitement ce document.

- Les exigences de la Loi sur les normes du travail relatives au certificat de travail semblent plus précises puisque dans ce cas, le certificat doit indiquer «le début et la fin de l'exercice des fonctions » alors que le Code ne traite que de la durée de l'emploi (ex.: Monsieur Brais fut à notre emploi pendant 3 ans).

- En vertu de la Loi sur les normes du travail, le certificat ne peut faire allusion à la qualité du travail ou encore, à la conduite du salarié. Cette modalité n'est pas reprise au Code. Peut-on en déduire que ce certificat pourrait contenir de telles mentions? Nous ne croyons pas que le Code civil interdise ces mentions dans la mesure où les deux parties y consentent. En effet, l'article 2096 C.c.Q. précise que le certificat fait état « uniquement » de la nature et de la durée de l'emploi et qu'à ce titre, l'employeur ne peut rien y ajouter sauf, selon nous, si

68 Il est également intéressant de comparer ces textes à celui de l'article L. 122-16, al. 1 du Code du travail français :

« L'employeur doit, à l'expiration du contrat de travail, délivrer au travailleur un certificat contenant exclusivement la date de son entrée et celle de sa sortie et la nature de l'emploi ou, le cas échéant, des emplois occupés successivement ainsi que les périodes pendant lesquelles les emplois ont été tenus. » 
l'employé y consent. Une telle dérogation serait permise en raison de 1'article 9 du Code qui édicte qu'il est possible de déroger aux règles du Code qui sont supplétives de volonté et qu'il ne s'agit pas, selon nous, d'une matière intéressant l'ordre public. Dans un tel cas, il y aurait là une distinction importante, pour ne pas dire une contradiction entre les deux textes législatifs. Ici encore, nous croyons que le texte de la Loi sur les normes du travail devrait prévaloir sauf naturellement dans les cas où la Loi sur les normes du travail ne peut recevoir application (ex. : un cadre supérieur ou un étudiant qui travaille au cours de l'année scolaire ${ }^{69}$ ).

- Ce certificat vise essentiellement à permettre au salarié de prouver à un employeur éventuel qu'il dispose d'une expérience professionnelle dans une entreprise pendant un certain temps, facilitant ainsi la recherche d'un emploi. Ȧ notre connaissance, aucune décision des tribunaux spécialisés ou de droit commun n'a traité de l'article 84 de la Loi sur les normes du travail. Est-ce à dire que ce certificat ne soulève aucune difficulté d'application? Nous l'ignorons. Cependant, nous croyons que son incorporation au contrat de travail pourrait, par exemple, donner lieu à une action en dommages-intérêts en cas de refus préjudiciable de la part de l'employeur de délivrer un tel certificat $^{\text {70 }}$. Les tribunaux français ont déjà reconnu la validité $\mathrm{d}^{\prime}$ 'une telle réclamation en dommages. Cependant, la législation française impose à l'employeur l'obligation de délivrer le certificat et au nouvel employeur celle de vérifier que le travailleur est bien libre de son dernier engagement. Car, le nouvel employeur est responsable civilement des conséquences d'embaucher un salarié déjà lié par un contrat de travail.

\section{L'ALIÉNATION D'ENTREPRISE}

Code civil du Québec

Art. 2097. L'aliénation de l'entreprise ou la modification de sa structure juridique par fusion ou autrement, ne met pas fin au contrat de travail.

Ce contrat lie l'ayant cause de l'employeur.
Loi sur les normes du travail

Art. 96. Aliénation d'entreprise. L'aliénation ou la concession totale ou partielle d'une entreprise autrement que par vente en justice $n$ 'invalide aucune réclamation civile qui découle de l'application de la présente

69 Voir art. 3 L.N.T.

70 Voir : G.H. CAmerYnCK, Gérard Lyon-Caen et Jean Pélissier, Droit du travail, Paris, Dalloz, 1984, p. 393 et suiv. 
loi ou d'un règlement et qui n'est pas payée au moment de cette aliénation ou concession. L'ancien employeur et le nouveau sont liés conjointement et solidairement à l'égard d'une telle réclamation.

Art. 97. Aliénation d'entreprise. L'aliénation ou la concession totale ou partielle de l'entreprise, la modification de sa structure juridique, notamment, par fusion, division ou autrement n'affecte pas la continuité de l'application des normes du travail.

L'article 2097 C.c.Q. constitue une innovation majeure qui aura une influence importante en droit du travail, qu'il suffise d'évoquer les points suivants :

- Avec cet ajout, la règle de la relativité des contrats est mise de côté. Le contrat de travail se rattache maintenant à l'entreprise et non au cocontractant ${ }^{71}$.

- Le libellé de l'article 2097 C.c.Q. nous semble suffisamment explicite pour considérer que la disposition est d'ordre public. D'ailleurs, l'article 45 C.t. dont le libellé est comparable, en ce sens qu'il n'est pas plus impératif, a toujours été interprété par la jurisprudence comme étant d'ordre public ${ }^{72}$. Ce caractère d'ordre public implique que les parties ne pourraient renoncer à l'avance à son application.

- L'art. 2097 C.c.Q. vient galvaniser les dernières failles des articles 45 et 46 du Code du travail. Il est maintenant clair qu'il y a transfert des contrats individuels de travail lors de la vente d'une entreprise.

- La définition retenue par le Code (art. 1525) pour circonscrire l'expression «constitue l'exploitation d'une entreprise » s'inscrit dans la logique de la décision de la Cour suprême dans l'arrêt Bibeault ${ }^{73}$.

- L'art. 2097 C.c.Q. ne fait pas d'exception pour la vente en justice, ce qui implique la subsistance du contrat de travail lors d'un tel type de vente ou encore, lors de la prise de possession des actifs par un syndic

71 Les tribunaux avaient reconnu que les articles 96 et 97 L.N.T. visaient à modifier le principe de la relativité des contrats (1022-1023 C.c.B.-C.); voir : Commission des normes du travail c. Foodcorp Ltd, [1988] R.J.Q. 2289 (C.P.).

72 Voir Charron Excavation Inc. c. Syndicat des employés de la ville de Ste-Thérèse, D.T.E. 89T-114 (T.T.), Transport de l'Anse Inc. c. Syndicat des chauffeurs d'autobus du Bas SaintLaurent (C.S.N.), [1986] T.T. 207.

73 U.E.S. local 298 c. Bibeault, [1988] 2 R.C.S. 1048. 
à la faillite. Cette solution est très différente de celle retenue par la jurisprudence dans le cadre de l'article 97 L.N.T. ${ }^{74}$.

- La disposition ne prévoit pas la possibilité d'une aliénation partielle de l'entreprise. Est-ce à dire que si l'employeur ne vend qu'une portion de son entreprise (ex. : $60 \%$ ), l'article 2097 C.c.Q. ne pourrait recevoir application? Une telle interprétation serait pour le moins incongrue, puisqu'il n'existe aucune raison pratique pour éviter l'application de la règle en matière d'aliénation partielle. De plus, le terme "aliénation » semble assez générique pour couvrir les deux réalités.

- Contrairement à l'art. 96 L.N.T., la disposition ne fait nullement mention de la concession de l'entreprise. Ce libellé fait en sorte que le Code civil ne protège pas le contrat de travail lors de l'octroi d'un contrat de sous-traitance. De la même manière, la survie du contrat de travail ne serait pas préservée lors de la prise de possession des actifs d'une entreprise par une institution financière ${ }^{75}$.

En ce qui a trait plus spécifiquement à la Loi sur les normes du travail, cette nouvelle disposition réglera la controverse qui consiste à savoir si les articles 96 et 97 de la Loi sur les normes du travail ont pour effet de transférer automatiquement les contrats de travail du vendeur à l'acquéreur d'une entreprise. Lors de la vente d'une entreprise, deux situations peuvent se produire. Premièrement, l'acquéreur, après la vente, continue à employer les salariés. Dans ce cas, la Cour d'appel dans l'arrêt Produits Pétro-Canada Inc. c. Émile Moalli et al. ${ }^{76}$ considère qu'il n'y a pas rupture de la notion de service continu. Cependant, la situation est différente lorsque le vendeur congédie les salariés lors de la vente et que l'acquéreur ne les réembauche pas. Dans ce dernier cas, la doctrine considère qu'il ne peut y avoir de lien de droit entre les salariés et l'acquéreur. Ainsi, lors de l'audition d'une plainte de congédiement sans cause juste et suffisante (art. 124 L.N.T.) l'acquéreur n'aurait pas à justifier le congédiement fait par le vendeur. Les auteurs Hébert et Trudeau s'expriment ainsi :

Avant de terminer cette discussion des articles 96 et 97, une mise en garde s'impose. Il ne faudrait pas voir dans ces seuls articles une forme de sécurité d'emploi lorsqu'un transfert d'entreprise s'effectue. L'employeur-cessionnaire demeure libre de prendre ou non les salariés de l'employeur-cédant à son service. Au plus peut-il être tenu responsable du préavis de l'article 82 en cas de défaut de

74 Bergeron c. Métallurgie Frontenac Ltée, D.T.E. 92T-1248 (C.A.).

75 À cet égard, les articles 96 et 97 L.N.T. peuvent recevoir application et constituent une meilleure protection pour le salarié : Commission des normes du travail c. Cie de gestion Welfab, [1989] R.J.Q. 2547 (C.S.); Perry c. Technologies Lanpor Inc., D.T.E. 91T-531 (C.T.).

76 [1987] R.J.Q. 261. Voir commentaires : Hélène OuIMET, « Commentaires sur l'affaire Produits Pétro-Canada c. Moalli », (1987) 47 R. du B. 852 et Fernand MORIN, Jurisprudence commentée en droit du travail, Cowansville, Les Éditions Yvon Blais Inc., 1992, p. 691. 
l'employeur-cédant. Pour qu'une telle dérogation au droit commun s'applique (soit l'obligation pour le nouvel employeur de garder les employés de l'ancien), il aurait fallu que le législateur utilise une formulation explicite à cet effet. Selon la forme actuelle de la loi, ce n'est que dans le cas où le cessionnaire garde à son emploi les salariés du cédant que la continuité de l'application des normes est assurée. Même l'existence du recours à l'encontre d'un congédiement sans cause juste et suffisante des articles 124 et suiv. ne change pas cet état de fait ${ }^{77}$.

Une décision de la Cour supérieure entérina ce point de vue ${ }^{78}$. Bien que la question soit litigieuse, avec la modification du Code civil, nous osons croire que le contrat de travail sera préservé et que l'acquéreur sera tenu de justifier le congédiement pratiqué par le vendeur. Ainsi, le salarié qui justifie de trois ans de service continu au sens de la Loi sur les normes du travail pourra faire valoir une plainte de congédiement sans cause juste et suffisante à l'encontre de l'ayant droit. En effet, puisqu'il existe dorénavant un fondement légal à la continuité du contrat de travail lors de l'aliénation d'une entreprise, il paraîtrait logique d'imposer à l'ayant cause l'obligation d'expliquer les motifs ayant entraîné le congédiement du salarié immédiatement avant la vente de l'entreprise. Une telle obligation imposée à l'acheteur est lourde de conséquences ${ }^{79}$, mais elle se dégage de l'esprit du nouveau Code qui impose aux parties l'obligation d'agir de bonne foi ${ }^{80}$ Cet ajout majeur permettra une plus grande protection du contrat de travail ${ }^{81}$. De plus, selon nous, la simple vente de l'entreprise ne pourra être considérée comme une cause juste et suffisante pour procéder au congédiement. En effet, il est difficile de prétendre que la vente d'une entreprise constitue en soi une cause juste et suffisante puisque si tel était le cas, l'article 2097 C.c.Q. perdrait toute application pratique. Ajoutons qu'au contraire, si le salarié ne justifie pas de trois ans de service continu, le vendeur peut congédier le salarié (art. 2091 C.c.Q.) et ce dernier ne pourra faire valoir aucun droit auprès de l'acheteur. D'ailleurs, ce dernier ne serait pas responsable du paiement du délai de congé dû au salarié en vertu de l'art. 2091 C.c.Q.

77 Gérard Hébert et Gilles Trudeau, Les normes minimales du travail au Canada et au Québec, Cowansville, Les Éditions Yvon Blais Inc., 1987, p. 56. Sur le même sujet voir : Georges AUDET et Robert BONHOMME, Le congédiement en droit québécois en matière de contrat individuel de travail, Cowansville, Les Éditions Yvons Blais Inc., 1988, p. 221-222.

78 Speer Canada (1988) Inc. c. Cloutier, D.T.E. 90T-1203 (C.S.); au même effet, voir : Commission des normes du travail c. Vêtements Victoriaville Inc., D.T.E. 88T-114 (C.P.); Commission des normes du travail c. Villa Notre-Dame de Lourdes et al., [1988] R.J.Q. 1965 (C.P.).

79 En effet, ce dernier pourrait être tenu de réintégrer le salarié congédié sans cause juste et suffisante.

80 Voir les articles 6,7 et 1375 C.c.Q.

81 On peut lire sur cette question: Jean-Yves Brière et Jean-Pierre VillaGGi, «Les normes du travail », Code du travail, Farnham, Les éditions CCH/FM (feuilles mobiles), 1992, p. 8,472 . 
puisqu'aucun droit de suite n'existe comme c'est le cas en vertu de l'article 96 L.N.T.

$\mathrm{Au}$ terme de notre démarche, il n'est pas inutile de tenter de tirer quelques conclusions d'ordre général :

- Il faut, du moins à ce stade de l'état du droit, éviter les jugements péremptoires car les tribunaux qui auront à trancher les multiples cas concrets qui se présenteront devront remplir une fonction normative de suppléance ou de complémentarité à une législation qui, à plusieurs égards, est lacunaire ${ }^{82}$.

- Dans un premier temps, il est clair que les tribunaux de droit commun ne pourront faire abstraction de la jurisprudence découlant de la Loi sur les normes pour interpréter le Code civil. La filiation entre certaines dispositions ne permettrait pas un écart considérable.

- L'harmonisation de certaines dispositions (ex. : art. 2094 C.c.Q.) avec d'autres contenues à la Loi sur les normes (ex. : art. 82.1) n'est pas évidente. Elle exigera des tribunaux un «effort » pour effectuer les conciliations nécessaires.

- L'apport d'une jurisprudence ayant interprété largement et libéralement la Loi sur les normes du travail aura certainement une influence sur l'interprétation du chapitre du Code civil portant sur le contrat de travail.

- Il est curieux que le législateur ait jugé à propos d'élever au stade de droit commun le certificat de travail qui, dans la réalité, n'a que très peu d'utilité.

- Le nouveau Code civil ne simplifiera pas la vie du justiciable puisqu'il n'unifie pas les droits et recours applicables en matière de relation de travail ${ }^{83}$ et particulièrement lors d'un congédiement.

En terminant, nous nous excusons du peu de réponses que nous pouvons fournir à nos interrogations. À ce propos, nous vous soumettons cette réflexion de Proust :

« Nous sentons très bien que notre sagesse commence où celle de l'auteur finit, et nous voudrions qu'il nous donnât des réponses, quand tout ce qu'il peut faire est de nous donner des désirs ${ }^{84}{ } »$.

82 En droit du travail, ce rôle a été maintes fois rempli par les tribunaux de droit commun. Sur cette question voir: Fermand MORIN, Jurisprudence commentée en droit du travail, Cowansville, Les Éditions Yvon Blais Inc., 1992, 860 p.

83 Pierre VERGE, «Faut-il nommer le contrat de travail? » (1988) 29 C. de D. 977.

84 Marcel Proust, Sur la lecture, Arles, Actes Sud, 1988, p. 32. 


\section{The Civil Code of Québec and the Labour Standards Act}

The purpose of the new Civil Code of Québec is to adapt the general law to the context of the Québec reality at the dawn of the twenty-first century. It is of the utmost importance for all citizens. Needless to say, the Code will directly affect labour legislation. The proposed analysis sets forth the consequences of the new Civil Code on the Labour Standards Act and, conversely, the incidence of the Labour Standards Act on the understanding of the applicable provisions of the Code. The analysis is both comparative and prospective. Six items are analysed for comparison purposes: the preeminence of the enactments, the concept of employee, the concept of continuous service, the breach of the employment relationship, the certificate of employment and the alienation of an undertaking.

The pre-eminence of the enactments. In case of insurmountable conflict, the Labour Standards Act should prevail over the general law as codified. Because the standards enacted are of necessary application, the Labour Standards Act shall apply notwithstanding the Civil Code of Québec.

The concept of employee. The two legislative enactments (art. 2085 C.C.Q. and s. 1(10) L.S.A.), if read together, show various similarities (requirement of masterservant relationship, no particular formality is required, forms of compensation are various, etc.). The two enactments contemplate the same reality. Moreover, the standards developed by the case law under the Labour Standards Act will be very useful for distinguishing between the employee and the independent contractor, as defined in the Civil Code.

The concept of continuous service. The analysis highlights the weak points resulting from the combined effect of arts. 2090 and 2091 C.C.Q. Thus, it may be possible for an employer to avoid the tacit renewal by granting a series of fixed term contracts. This way, the employer would not be bound to pay an indemnity in lieu of a notice of termination in case of termination. This solution chosen by the legislator is hardly compatible with the letter, the meaning and the spirit of s. 124 L.S.A.

Breach of the employment relationship. Under this heading, the comparison of the applicable sections raises some questions :

- How to reconcile the serious reason specified in article 2094 C.C.Q. and the concept of serious fault (s. 82.1 L.S.A.)?

- How to reconcile the serious reason and the concept of good and sufficient cause (s. 124 L.S.A.)?

- Does the payment of an indemnity by the employer pursuant to s. 82 L.S.A. exclude a claim for a notice of termination based on art. 2091 C.C.Q.?

The certificate of employment. The Civil Code states in art. 2096 that upon termination of an employment contract, the employer shall provide the employee with a certificate of employment. Such a certificate is also required under the Labour Standards Act. Here again this doubling raises questions : 
- Is it possible to add comments on the quality of work done by the employee?

- Which consequences result from the failure to provide this document?

Alienation of an undertaking. Article 2097 of the Civil Code supplements ss. 96 and 97 L.S.A. by protecting the employment contract. It will now be possible to argue that the purchaser of an undertaking will be bound to explain the grounds justifying a dismissal which occurred immediately before the sale of the undertaking. Such a proof will be made in the context of a complaint of dismissal without a good and sufficient cause (s. 124 L.S.A.).

From this analysis, the author draws certain conclusions :

- Case law relating to the Labour Standards Act will be very useful to the interpretation of the Civil Code. The links between certain provisions justifies this approach.

- The relationship of some provisions (art. 2094 C.C.Q.) with others contained in the Labour Standards Act (s. 82.1) is not clear.

- The contribution of case law which provides a liberal and extensive interpretation of the Labour Standards Act will likely have a material impact on the interpretation of the chapter of the Civil Code relating to the employment contract.

- The new Code does not lighten the burden of the citizen in case of dismissal. It would have been desirable to unify the applicable rights and remedies.

Actes du colloque du 6 octobre 1993 sous l'égide du Conseil consultatif du travail et de la main-d'œuvre et du Département des relations industrielles de l'Université Laval

\section{Le Code civil et les relations du travail}

COLLECTION INSTRUMENTS DE TRAVAIL Département des relations industrielles, Université Laval

Services des ventes

Les Presses de l'Université Laval

Cité universitaire, Sainte-Foy

Québec, Canada, G1K 7P4

tél. : (418) 656-5106

téléc. (418) 656-3476

ISBN 2-920259-36-9 - 1993 - 114 p. - \$ 12. 